\title{
Non-linear signaling for pattern formation? Ben Scheres
}

\begin{abstract}
Developmental pathways are often simplified as linear cascades of gene activation caused by initial signaling events. Recent data, however, support a more complex view in which non-linear responses to signals are important for pattern formation. First, a positive feedback loop may regulate cellular patterning in the Arabidopsis epidermis. Second, an asymmetric auxin distribution that organizes pattern in the distal root tip may be established by positive feedback.
\end{abstract}

\section{Addresses}

Department of Molecular Cell Biology, Utrecht University, Padualaan 8, 3584 CH Utrecht, The Netherlands; e-mail: b.scheres@bio.uu.nl

\section{Current Opinion in Plant Biology 2000, 3:412-417}

$1369-5266 / 00 / \$$ - see front matter

(C) 2000 Elsevier Science Ltd. All rights reserved.

$\begin{array}{ll}\text { Abbreviations } \\ \text { ARF } & \text { auxin response factor } \\ \text { axr6 } & \text { auxin-resistant } 6 \\ \text { bdl } & \text { bodenlos } \\ \text { bHLH } & \text { basic helix-loop-helix } \\ \text { CPC } & \text { CAPRICE } \\ \text { gl1 } & \text { glabrous1 } \\ \text { gl2 } & \text { glabra } 2 \\ \text { MP } & \text { MONOPTEROS } \\ \text { PIN1 } & \text { PIN-FORMED 1 } \\ \text { QC } & \text { quiescent center } \\ \text { TRY } & \text { TRIPTYCHON } \\ \text { ttg } & \text { transparent testa glabra } \\ \text { WER } & \text { WEREWOLF }\end{array}$

\section{Introduction}

Many cell lineage studies in plants have clarified that cells differentiate according to their position within the plant [1]; but how are cells informed about their position? Do receptor-ligand interactions, and in some cases transcription factors, signal the position of cells and instruct cell fate accordingly? Is the initial asymmetric distribution of signals critical for pattern formation, as it is in animal systems? Receptor kinase-mediated signaling in the shoot apical meristem has indeed been shown to provide spatial information within fields of cells $\left[2,3^{\bullet \bullet}\right]$, and a combination of transcription factors is known to specify organ primordia in flowers [4], but how spatial information is set up has remained unresolved.

This review discusses the generation of two cellular patterns in Arabidopsis: first, hair cells interspersed with non-hair cells in the epidermis, and second, quiescent center and root-cap cells in the distal root tip. Recent studies have identified molecules that may act as positional signals and have begun to emphasize non-linear mechanisms that establish their asymmetric distribution.

\section{Cell patterning of epidermal hairs}

When they can first be recognized, the hair-bearing cells of the leaf epidermis (i.e. trichomes) are separated by three or four epidermal cells (Figure 1a). As reflected in their names, glabrous1 (gl1), transparent testa glabra (ttg) and glabra2 (gl2) mutants are defective in trichome development [5,6]. GL1 and GL2 encode myb and homeobox transcription factors that are expressed in trichome cells $[7,8]$ (Figure 1b). TTG encodes a small WD40-repeat protein that resembles a $\mathrm{G}-\beta$ subunit $\left[9^{\bullet}\right]$.

Two observations suggest that trichome patterning involves cell-cell communication within the epidermal cell layer. First, their spacing bears no obvious references to underlying tissues. Second, analysis of genetically marked sectors reveals no systematic division patterns to generate trichomes and surrounding epidermal cells [10]. The occurrence of trichome clusters in certain mutant combinations, but not in the wild-type, suggests that regulation of trichome spacing involves lateral inhibition, that is, the suppression of trichome fate in cells neighboring trichome precursor cells. Analysis of genetic combinations that promote trichome clustering has shown that three genes are involved in trichome spacing. Mutations in the TRIPTYCHON (TRY) gene, weak ttg mutants, and overexpression of the GL1 gene in certain $t t g$ heterozygotes result in trichome clusters [6,11]. Thus, activity levels of the TRY, GL1 and $T T G$ genes appear to be important for trichome spacing.

A recent study demonstrates that try clusters can consist of cells with different ancestry, thus TRY acts nonautonomously and inhibits trichome fate in neighboring cells [12••]. Overexpression of GL1 dramatically increases cluster formation in try mutants only, suggesting that TRY prevents trichome clustering by suppression of GL1 function (Figure 1c). Overexpression of the maize $R$ gene in the try background also increases cluster formation, suggesting that $T R Y$ suppresses $T T G$ function $-R$ rescues $t$ tg mutants and encodes a basic helix-loop-helix (bHLH) protein that is thought to act downstream of TTG (Figure 1d) [13]. TTG appears not only to be a target for $T R Y$-mediated inhibition but also to contribute to this inhibition, because several $t r y /+t t g /+$ heterozygotes display increased cluster formation compared to the single heterozygotes. A positive feedback loop can be inferred from genetic combinations with differently modulated TTG and GL1 activities, which place TTG either upstream or downstream of GL1. In the resulting model, the activators $T T G$ and GL1 stimulate their own production and that of the non-autonomous inhibitor TRY thereby amplifying initial stochastic differences and stabilizing cell-fate choice [12 ${ }^{\bullet \bullet}$ ] (Figure 1c,d). This attractive mutual inhibition model of pattern formation derives in part from ubiquitous expression experiments that do not directly show in which cells and at which stage the overexpressed genes influence patterning. Specific promoters and clonal expression systems can now be used in further tests. 


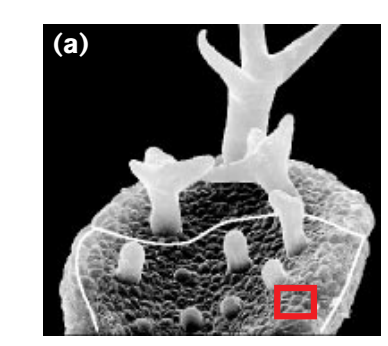

(e)

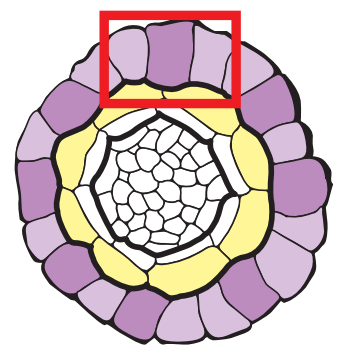

(b)

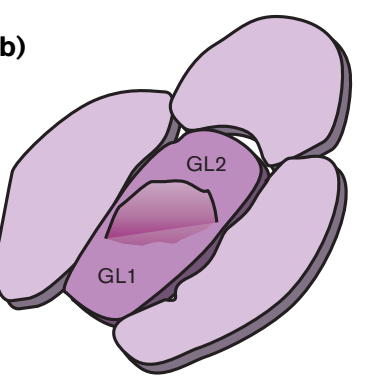

(f)

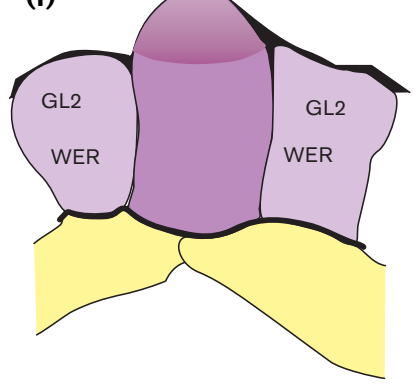

(c)

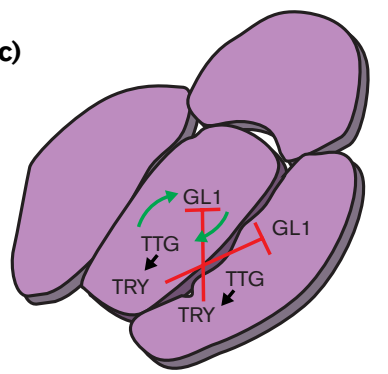

(g)

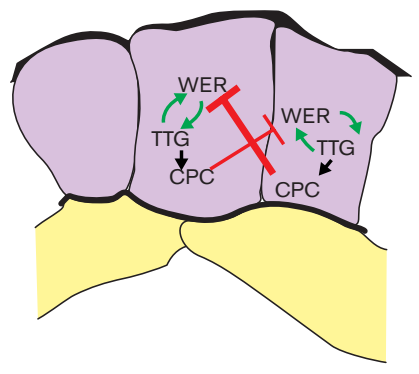

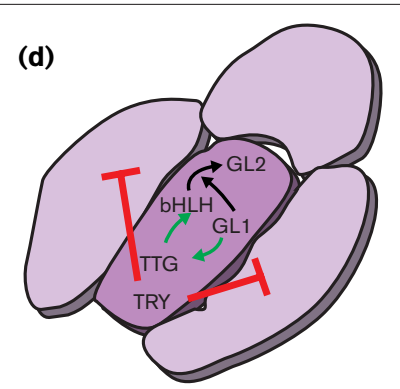

(h)

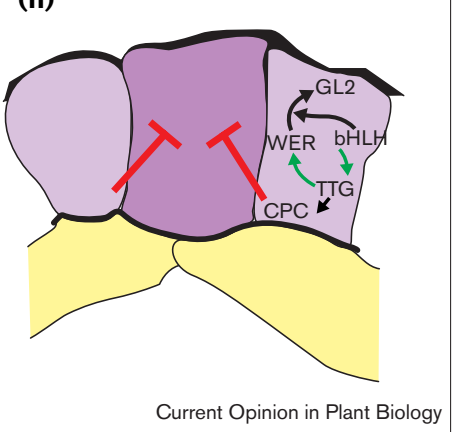

Models for epidermal hair patterning in Arabidopsis. (a-d) Trichomes. (a) Trichomes develop from immature leaf epidermis with regular spacing intervals, which have been shown not to be correlated to cell lineage (image kindly provided by Martin Hülskamp). (b) At the stage of hair outgrowth, GL1 and GL2 transcription factors are restricted to incipient trichomes (dark purple). (c) Two subsequent stages of trichome selection. A positive feedback loop between TTG and GL1 (green arrows) is coupled to the production of TRY, which acts as a non-cell-autonomous inhibitor (red bars). (d) This system amplifies initially small differences in activity into a stable pattern of trichome precursor cells that inhibit trichome fate in neighbor cells. (e-h) Trichoblasts. (e) Incipient root hair cells (dark purple) overlie a cortical cell wall. (f) At the stage of outgrowth, the non-hair cells express WER and GL2 transcription factors. (g,h) Two subsequent stages of non-hair cell selection. Unknown positional cues provide an activity bias for a positive feedback loop between TTG and WER (green arrows) coupled to CPC, which acts as non-autonomous inhibitor (red bars). The biasing signal favors the emergence of hair cells over the cortical cell wall. Dark purple shading indicates commitment to $(b-d)$ trichome and $(f-h)$ atrichoblast fate.
In Arabidopsis roots, files of epidermal cells that overlie more than one underlying cortex cell develop hairs. Files with hair-bearing cells (i.e. trichoblasts) are interspersed with files of hairless cells (i.e. atrichoblasts) (Figure 1e) [14]. Epidermal cells in the root meristem occasionally generate daughter cells that become re-allocated with respect to the underlying cortex. These cells switch fate as evidenced by both morphological and gene expression criteria [15]. Hence, the cortical cell layer appears to convey positional information for epidermal cell fate.

Both $t t g$ and $g l 2$ mutants display an excess of hair-bearing cells in the root epidermis at the position where non-hair cells are expected; conversely, they have an excess of hairless cells in the shoot epidermis [16,17]. GL2 is expressed in the hairless cells, suggesting that it is required cell-autonomously for the hairless cell fate (Figure 1f) [17]. The maize $R$ gene can suppress hair-cell fate in the $t$ tg mutant background, suggesting that TTG also activates an Arabidopsis R-like bHLH protein in the root (Figure 1h) [16].

Two genes have been identified in which loss-of-function mutations affect only root epidermal patterning.
The CAPRICE $(C P G)$ gene, encoding a small protein with a MYB-like DNA-binding domain but without recognizable activator domains, was identified as a negative regulator of $G L 2$; this regulatory role is consistent with the presence of excess non-hair cells in $c p c$ mutants [18]. The WEREWOLF (WER) myb gene is required for the hairless fate in roots [19 $\left.{ }^{\bullet}\right]$. WER is expressed in non-hair cells, is homologous to GL1 and acts (as does GL1 in the leaf epidermis) as an upstream regulator of GL2 expression (Figure 1h). WER can also interact with $\mathrm{R}$, and hence (like GL1 in the leaf) potentially links TTG input to GL2 expression.

The identification of the WER gene as a GL1 homologue and the related functions of TTG and GL2 in both root and shoot epidermis point to the involvement of a similar mutual inhibition network in patterning the root and shoot epidermis. In both the root and shoot epidermis, a TTGregulated bHLH protein and a MYB factor (i.e. GL1 in shoots and WER in roots) are involved in the specification of one cell type. In trichomes, TRY is the non-autonomous inhibitor, and in atrichoblasts the CPC gene product is a candidate inhibitor. CPC is mostly expressed in non-hair cells (T Wada, K Okada, personal communication) and its 
removal reduces the number of hair cells, consistent with a function as a non-cell-autonomous suppressor of non-hair cell fate (Figure 1g). It is tempting to speculate that TRY and CPC perform related functions and that they could be related molecules. In a simple hypothetical scenario, inhibitors, such as TRY and CPC, might move directly to the target cell where they would compete with the GL1 and WER activator MYBs for binding sites (Figure 1c and f). Plasmodesmatal transport of proteins might enable this movement [20], but were this to be the case, the lack of inhibitory action of TRY and CPC in the protein-producing cell should be explained. Alternatively, TRY and CPC may act indirectly by regulating the production of as yet unidentified signals.

Whereas the shoot epidermal patterning system seems to generate pattern de novo from initial stochastic fluctuations, root epidermal cells appear to respond to cues from underlying cortical cells. The underlying mechanism in the shoot and roots might be similar if the cortex produces a localized, possibly cell-wall derived [15], signal that biases the activity of a stochastic patterning mechanism through interaction with one of its components. In the root epidermis, such a biasing mechanism could ensure the formation of continuous files of trichoblasts and non-trichoblasts.

\section{Vascular and distal root patterning}

The phytohormone auxin induces linear vascular strands upon external application. A non-linear feed-forward mechanism termed 'canalization' has been proposed to account for this ability. In this scenario, cells with elevated auxin levels become better conduits and therefore channel auxin to their basal end, leading to elevated auxin concentrations in their basal neighbor cell [21]. The model predicts that auxin accumulation influences flux, and flux influences accumulation, forming a self-organizing positive feedback loop that acts as a patterning system. The reported effects of polar auxin transport inhibition on vascular strand formation in Arabidopsis leaves are consistent with the canalization model [22 $\left.2^{\bullet}\right]$.

The PIN-FORMED1 (PIN1) and EIR1/PIN2 proteins, which are thought to be components of polar auxin efflux carriers, are candidate components of the proposed positive feedback system [23,24]. Intriguingly, the GNOM/EMB30 gene that encodes a guanosine nucleotide exchange factor for small $\mathrm{G}$-proteins involved in vesicle formation, is required for the coordinated polar localization of at least one PIN family member in embryonic cells $\left[25^{\bullet}\right]$. This study further demonstrates that the polar localization of PIN1 involves a dynamic equilibrium that might respond to regulatory factors during patterning.

The MONOPTEROS (MP) gene, which is required for the correct formation of a continuous vascular system, encodes a member of the auxin response factor (ARF) protein family $[26,27]$. ARF transcription factors bind to, and regulate transcription of, auxin responsive promoters [28]. These findings support a prominent role for auxin in vascular patterning. Interestingly, the recently described auxin-resistant 6 (axrb) mutants display phenotypes similar to those of $m p$ mutants [29••]. In addition, axr6 heterozygotes are auxin resistant. Functional analysis of the PIN proteins MP and AXR6, and their interacting factors, should now enable molecular testing of the canalization hypothesis.

Vascular strand formation is not the only patterning process that depends on auxin. The promoting effect of auxin on root formation has been well-established from tissue culture experiments. The finding that $m p$ and axr 6 mutants, as well as the recessive auxin-resistant bodenlos ( $b d l)$ mutation $\left[30^{\bullet \bullet}\right.$, lack the entire root suggests that this promoting effect has an early role in normal root development. Consistent with this idea, the application of auxin transport inhibitors or auxin analogs to explanted Brassica juncae embryos produces root defects [31].

The root tip of Arabidopsis contains three distal-specific cell types, the columella- and lateral-root-cap cells, and the quiescent center (QC) cells [14] (Figure 2a). Cell identity in the root cap and the $\mathrm{QC}$ region depends primarily on position [32], and recent evidence suggests that auxin is the relevant positional cue. From the heart stage of embryogenesis onwards, the local activity of a synthetic auxin-response element indicates that maximum auxin response is associated with the distal root tip [33*0] (Figure 2b). Auxin-resistant axr1 and axr3 mutants [34,35] reduce the response maximum and show correlated defects in the specification or arrangement of distal cell types. These findings suggest that the AXR1 and AXR3 genes are required for the perception of a distal auxin maximum and for distal patterning [33••]. In pin1 and pin2/eir1 mutants, which are thought to be primarily defective in vascular and peripheral polar auxin transport, respectively $[23,24,36]$, the localization of this auxin maximum is less precise, and is correlated with mild defects in the orientation of cell division and in cell elongation in the distal root region. Thus, PIN genes appear to have a role in localization of the auxin maximum. The importance of $P I N$ genes can now be assessed by the systematic analysis of this large gene family. A significant shift in the auxin maximum after application of polar auxin transport inhibitors corroborates the importance of polar transport for the generation of asymmetric auxin distribution in the root tip. Dramatic re-specification of distal cell types upon auxin transport inhibition suggests that the auxin maximum is sufficient for specifying the location of distal cell

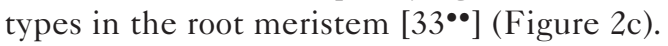

It can be questioned whether the local auxin concentration threshold, which acts as a spatial cue for pattern formation in the root tip, is set up using positive feedback along the lines of the canalization hypothesis. After inhibition of polar auxin transport and a shift in auxin distribution, root cells adjust cell-division planes to the new position of the maximum [33 $\left.{ }^{\bullet}\right]$. Moreover, inverse 


\section{Figure 2}

Root patterning in Arabidopsis. (a) The distal root contains three specific cell types: columella root-cap cells, lateral root-cap cells and quiescent center cells. (b) The DR5::GUS auxin-response reporter detects a distal maximum in the columella initial cells. (c) Displacement of the auxin (reporter) concentration maximum, in this case by polar auxin transport inhibition, leads to dramatic re-patterning. $(\mathbf{d}-\mathbf{f})$ A model that depicts the generation of an auxin threshold that organizes pattern in three stages. In analogy with the canalization hypothesis, initially small differences in the net cellular direction of auxin transport (slightly biased towards the basal end) generate and stabilize an auxin maximum through positive feedback between auxin concentration and the positioning of auxin transporters.

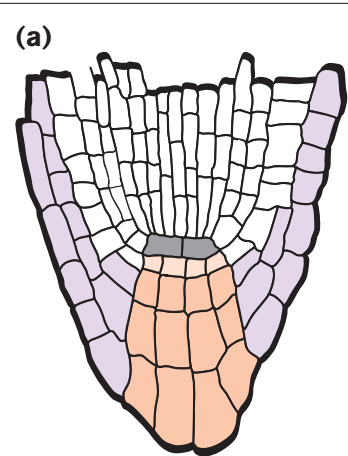

(d)

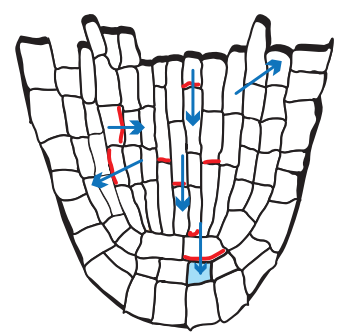

(b)

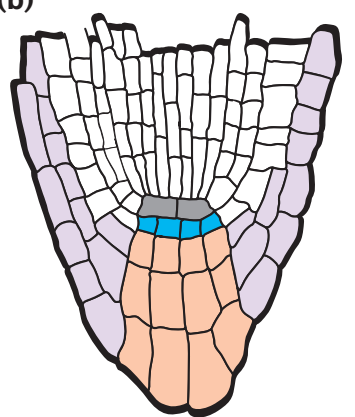

(e)

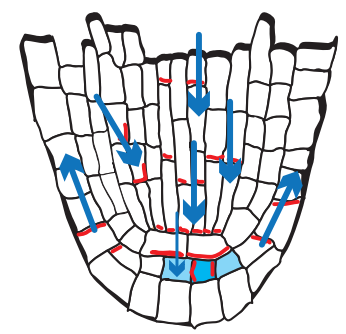

(c)

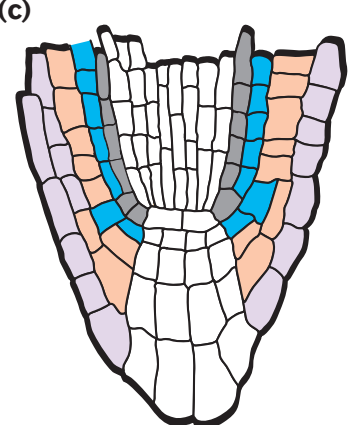

(f)

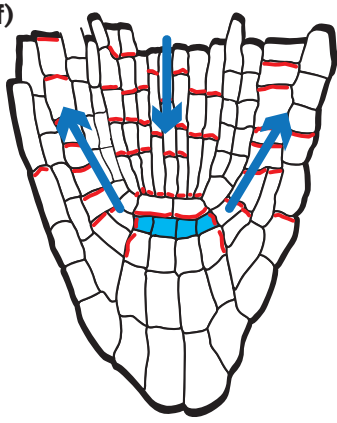

Lateral root cap

Quiescent center

Columella root cap

Columella initials
Auxin (reporter) maximum

- Auxin efflux carrier

$\longrightarrow$ Auxin transport sites of root-hair emergence on epidermal cells at opposite sites of an auxin maximum suggest cell polarity reversal. Thus, cell polarity seems to be dependent on auxin distribution, and changed polarity, in turn, might feed back on auxin distribution and the location of a maximum. Such a feedback mechanism could act, at least in part, through auxin-dependent positioning of transporters (Figure 2d-f). In conclusion, patterning may involve a positive feedback loop, in which transporters determine auxin localization, and localized auxin reinforces transporter location (Figure 2d-f). Such a mechanism could be stochastic in principle, in analogy to the proposed trichome patterning system, but biased, in analogy to the proposed mechanism of trichoblast patterning, to give a maximum at the basal embryo region during normal development. Perhaps such a stochastic component underlies the auxin-induced root initiation process in tissue culture [37].

\section{Conclusions}

Recent analyses of pattern formation in the Arabidopsis epidermis and distal root tip suggest that feedback loops play a role in the generation of positional information. Although the systems discussed here point to the involvement of non-linear mechanisms in the generation of patterning information, other examples exist in which nonlinear signaling contributes to the maintenance of patterning information $\left[3^{\bullet \bullet}, 38^{\bullet \bullet}, 39\right]$. In the epidermis, where the genetic evidence for non-linear relationships is strongest, much of the proposed feedback takes place in single cells, and cell biological and biochemical approaches will be needed to verify the proposed interactions. In the root tip, the genetic evidence for non-linearity is just emanating, but the intercellular nature of the suspected feedback in this organ may facilitate the molecular-genetic dissection of proposed regulatory interactions.

Even when the components of feedback mechanisms become known, non-linear mechanisms are not simply intuitive and quantitative analysis of regulatory factors by mathematical modeling might become essential to understanding patterning at a deeper level. In this vein, an exciting analogy exists between the generation of stable spatial patterns from initially small differences in plant development and non-linear differential equations where different initial conditions lead to the same 'attractor' state. Do pattern 'attractors' explain some of the mysterious flexibility of plant development, and do we need to dig out our math books to improve our understanding? 


\section{Acknowledgements}

I would like to thank Markus Grebe for helpful discussions on this manuscript, and Martin Hülskamp for providing the scanning electron microscope image used in Figure 1.

\section{References and recommended reading}

Papers of particular interest, published within the annual period of review, have been highlighted as:

- of special interest

- of outstanding interest

1. Steeves TA, Sussex IM: Patterns in plant development. Cambridge, Cambridge University Press; 1989.

2. Clark SE, Williams RW, Meyerowitz EM: The CLAVATA1 gene encodes a putative receptor kinase that controls shoot and floral meristem size in Arabidopsis. Cell 1997, 89:575-585.

3. Fletcher JC, Brand U, Running MP, Simon R, Meyerowitz EM:

- Signaling of cell fate decisions by CLAVATA3 in Arabidopsis shoot meristems. Science 1999, 283:1911-1914.

This study of the molecular identity and expression of the CLV3 gene proposes a feedback model between CLV1 and CLV3 expressing cells that maintains shoot meristem size.

4. Weigel D, Meyerowitz EM: The ABCs of floral homeotic genes. Cell 1994, 78:203-209.

5. Koornneef M, van Eden J, Hanhart CJ, Stam P, Braaksma FJ, Feenstra WJ: Linkage map of Arabidopsis thaliana. J Hered 1983 74:265-272

6. Hülskamp M, Misra $S$, Jürgens $G$ : Genetic dissection of trichome cell development in Arabidopsis. Cell 1994, 76:555-566.

7. Oppenheimer DG, Herman PL, Esch J, Sivakumaran S, Marks MD: A myb-related gene required for leaf trichome differentiation in Arabidopsis is expressed in stipules. Cell 1991, 67:483-493.

8. Rerie WG, Feldmann KA, Marks MD: The GLABRA2 gene encodes a homeodomain protein required for normal trichome development in Arabidopsis. Genes Dev 1994, 8:1388-1399.

9. Walker AR, Davison PA, Bolognesi-Winfield AC, James CN

- Srinivasan N, Blundell TL, Esch JJ, Marks MD, Gray JC: The TRANSPARENT TESTA GLABRA1 locus, which regulates trichome differentiation and anthocyanin biosynthesis in Arabidopsis, encodes a WD40 repeat protein. Plant Cell 1999, 11:1337-1350.

In this paper, the molecular cloning of TTG reveals that it encodes a protein containing four WD40-repeats, motifs that are found in signaling proteins. The findings are consistent with a role for TTG as an upstream regulator of the MYC/MYB-like gene products involved in epidermal patterning.

10. Larkin JC, Young N, Prigge M, Marks MD: The control of trichome spacing and number in Arabidopsis. Development 1996, 122:997-1005.

11. Larkin JC, Oppenheimer DG, Pollock S, Marks MD: The roles of the GLABROUS1 and TRANSPARENT TESTA GLABRA genes in Arabidopsis trichome development. Plant Cell 1994, 6:1065-1076.

12. Schnittger $A$, Folkers $U$, Schwab $B$, Jürgens $G$, Hülskamp $M$ :

-. Generation of a spacing pattern: the role of TRIPTYCHON in trichome patterning in Arabidopsis. Plant Cell 1999, 11:1105-1116.

Arabidopsis plants containing different combinations of try, ttg and g/1 alleles that overexpress $G L 1$ and $R$ (a transcriptional regulator downstream of TTG) are analyzed. The results, and additional clonal analysis, suggest that a local self-amplifying activator loop in incipient trichomes, involving $G L 1$ and $T T G$, induces $T R Y$ as a lateral inhibitor of trichome fate.

13. Lloyd AM, Walbot V, Davis RW: Anthocyanin production in dicots activated by maize anthocyanin-specific regulators $\mathrm{R}$ and $\mathrm{C} 1$. Science 1992, 258:1773-1775.

14. Dolan L, Janmaat K, Willemsen V, Linstead P, Poethig S, Roberts K, Scheres B: Cellular organisation of the Arabidopsis root. Development 1993, 119:71-84.

15. Berger F, Haseloff J, Schiefelbein J, Dolan L: Positional information in root epidermis is defined during embryogenesis and acts in domains with strict boundaries. Curr Biol 1998, 8:421-430.

16. Galway ME, Masucci JD, Lloyd AM, Walbot V, Davis RW, Schiefelbein JW: The TTG gene is required to specify epidermal cell fate and cell patterning in the Arabidopsis root. Dev Biol $1994,166: 740-754$
17. Masucci JD, Rerie WG, Foreman DR, Zhang M, Galway ME, Marks MD, Schiefelbein JW: The homeobox gene GLABRA 2 is required for position-dependent cell differentiation in the root epidermis of Arabidopsis thaliana. Development 1996, 122:1253-1260.

18. Wada T, Tachibana T, Shimura Y, Okada K: Epidermal cell differentiation in Arabidopsis determined by a Myb homolog, CPC. Science 1997, 277:1113-1116.

19. Lee MM, Schiefelbein J: WEREWOLF, a MYB-related protein in -. Arabidopsis, is a position-dependent regulator of epidermal cell patterning. Cell 1999, 99:473-483.

The authors show that wer mutants are defective in specification of the nonhair cells in the root epidermis. The WER gene encodes a MYB protein Epistasis and yeast two-hybrid analyses suggest that WER cooperates with an Arabidopsis homologue of the maize transcriptional activator $\mathrm{R}$ as a positive regulator of GL2 to specify the non-hair fate.

20. Lucas WJ, Bouche-Pillon S, Jackson DP, Nguyen L, Baker L, Ding B Hake S: Selective trafficking of KNOTTED1 homeodomain protein and its mRNA through plasmodesmata. Science 1995, 270:1980-1983.

21. Sachs T: Cell polarity and tissue patterning in plants. Development 1991 (suppl 1):83-93.

22. Mattsson J, Sung ZR, Berleth T: Responses of plant vascular

-. $\quad$ systems to auxin transport inhibition. Development 1999, 126:2979-2991.

Several auxin transport inhibitors are shown to evoke stage-dependent defects in vascular differentiation and vascular strand alignment during leaf development. These effects are correlated with anatomical stages of of vascular development in early leaf primordia. These findings suggest that auxin transport is required for vascular tissue continuity and the restriction of vascular development to narrow strands.

23. Gälweiler L, Guan C, Müller A, Wisman E, Mendgen K, Yephremov A, Palme K: Regulation of polar auxin transport by AtPIN1 in Arabidopsis vascular tissue. Science 1998, 282:2226-2229.

24. Muller A, Guan C, Gälweiler L, Tanzler P, Huijser P, Marchant A Parry G, Bennett M, Wisman E, Palme K: AtPIN2 defines a locus of Arabidopsis for root gravitropism control. EMBO J 1998 , 17:6903-6911.

25. Steinman T, Geldner N, Grebe M, Mangold S, Jackson CL, Paris S,

-• Gälweiler L, Palme K, Jürgens G: Coordinated polar localization of auxin efflux carrier PIN1 by GNOM ARF GEF. Science 1999, 286:316-318.

The failure of gnom mutants to consistently localize PIN1, a component of an auxin efflux carrier, may explain aspects of gnom polarity defects in terms of altered auxin distribution. The GNOM protein displays Brefeldin A (BFA)sensitive guanosine exchange activity on mammalian ARF, and co-purifies with membranes in a BFA-dependent fashion, consistent with its playing a role in protein trafficking.

26. Berleth $\mathrm{T}$, Jürgens $\mathrm{G}$ : The role of the monopteros gene in organising the basal body region of the Arabidopsis embryo. Development 1993, 118:575-587.

27. Hardtke CS, Berleth T: The Arabidopsis gene MONOPTEROS encodes a transcription factor mediating embryo axis formation and vascular development. EMBO J 1998, 17:1405-1411.

28. Ulmasov T, Hagen G, Guilfoyle TJ: ARF1, a transcription factor that binds to auxin response elements. Science 1997, 76:1865-1868.

29. Hobbie L, McGovern M, Hurwitz LR, Pierro A, Liu NY,

-. Bandyopadhyay A, Estelle M: The axr6 mutants of Arabidopsis thaliana define a gene involved in auxin response and early development. Development 2000, 127:23-32.

The authors show that axr6 homozygotes are defective in embryonic development, with alterations that strikingly resemble those described for $m p$ mutants. These heterozygotes are also auxin-insensitive when two wildtype AXR3 copies are present, suggesting that the axr6 alleles are gainof-function mutants and that auxin perception has a role in vascular and embryonic patterning.

30. Hamann T, Mayer U, Jürgens G: The auxin-insensitive bodenlos

-. mutation affects primary root formation and apical-basal patterning in the Arabidopsis embryo. Development 1999 , 126:1387-1395.

A careful description of the development of the $b d /$ mutant, its genetic interaction with axr1 mutants and its reduced sensitivity to auxin provides evidence that auxin perception is important for correct apical-basal patterning from the early stages of embryogenesis onward. 
31. Hadfi K, Speth V, Neuhaus G: Auxin-induced developmenta patterns in Brassica juncea embryos. Development 1998 125:879-887.

32. Van den Berg C, Willemsen V, Hage W, Weisbeek P, Scheres B: Cell fate in the Arabidopsis root meristem determined by directional signalling. Nature 1995, 378:62-65.

33. Sabatini S, Beis D, Wolkenfelt H, Murfett J, Guilfoyle T, Malamy J,

- Benfey P, Leyser O, Bechtold N, Weisbeek P, Scheres B: An auxindependent distal organizer of pattern and polarity in the Arabidopsis root. Cell 1999, 99:463-472.

A GUS reporter driven by a synthetic auxin response element displays a concentration maximum in the distal root tip. This maximum is affected in the auxin response mutants axr1 and axr3, and in pin mutants (which have defective polar auxin transport), correlating with their patterning defects. Induction of an ectopic maximum by polar auxin transport inhibitors, auxin addition or laser ablation causes re-specification of distal cell types and polarity shifts.

34. Leyser O, Lincoln CA, Timpte C, Lammer D, Turner J, Estelle M Arabidopsis auxin-resistance gene AXR1 encodes a protein related to ubiquitin-activating enzyme E1. Nature 1993, 364:161-164
35. Rouse D, Mackay $\mathrm{P}$, Stirnberg $\mathrm{P}$, Estelle $\mathrm{M}$, Leyser O: Changes in auxin response from mutations in an $A U X / I A A$ gene. Science 1998, 279:1371-1373.

36. Luschnig C, Gaxiola RA, Grisafi P, Fink GR: EIR1, a root-specific protein involved in auxin transport, is required for gravitropism in Arabidopsis thaliana. Genes Dev 1998, 12:2175-2187.

37. Skoog F, Miller CO: Chemical regulation of growth and organ formation in plant tissues cultured in vitro. Symp Soc Exp Biol 1957, 11:118-131.

38. Schoof $H$, Lenhard M, Haeker A, Mayer KFX, Jürgens G, Laux T: The

- stem cell population of Arabidopsis shoot meristems is maintained by a regulatory loop between the CLAVATA and WUSCHEL genes. Cell 2000, 100:635-644.

Evidence is presented for the existence of a signaling feedback loop, involving the CLAVATA1 transmembrane receptor kinase pathway and cells specified by the WUSCHEL homeodomain protein, that maintains the size of the stem-cell region in the shoot apical meristem of Arabidopsis.

39. Lee JJ, von Kessler DP, Parks S, Beachy PA: Secretion and localized transcription suggest a role in positional signaling for products of the segmentation gene hedgehog. Cell 1992, 71:33-50. 